\title{
Sintomas depressivos em gestantes da atenção básica: prevalência e fatores associados
}

\author{
Depressive symptoms in primary care pregnant women: prevalence and \\ associated factors
}

Rafaela Santi Dell'Osbel'1, Maria Luisa de Oliveira Gregoletto², Cleber Cremonese ${ }^{3}$

${ }^{1}$ Curso de Nutrição, Centro Universitário da Serra Gaúcha (FSG) - Caxias do SUL (RS), Brasil.

2Programa de Pós-graduação em Saúde Coletiva, (FSG) - Caxias do SUL (RS), Brasil.

${ }^{3}$ Departamento de Saúde Pública e Meio Ambiente. (FSG) - Caxias do SUL (RS), Brasil.

DOI: https://dx.doi.org/10.7322/abcshs.v44i3.1241

\section{RESUMO}

Introdução: O aparecimento de transtornos emocionais no início da gestação é frequente, entre eles estão os sintomas depressivos (SD), como sentimento de culpa, falta de apetite e de energia. Objetivo: Medir a prevalência de sintomas depressivos e fatores associados em gestantes atendidas na Atenção Básica. Métodos: Estudo epidemiológico observacional transversal, constituído por gestantes, usuárias da Atenção Básica de Caxias do Sul/RS. Os SD foram avaliados por meio Patient Health Questionnaire (PHQ9). Realizou-se a análise bruta, onde calculou-se as razões de prevalência (RP) e respectivos intervalos de confiança (IC 95\%). A comparação de variáveis categóricas ocorreu por meio do teste de Qui-Quadrado. A análise ajustada foi realizada por regressão de Poisson, utilizou-se a técnica de backwards, onde o modelo final foi construído a partir das variáveis com $p \leq 0,20$ na análise bruta. Resultados: A amostra constituiu-se de 76 gestantes, destas 46,1\% apresentaram SD. A média de idade foi de 26,6 anos $( \pm 5,95)$ e $72,4 \%$ estavam casadas ou em união estável. Houve associação significativa entre SD e estado civil (RP: 1,54; IC 95\% 1,00-2,37; $p=0,045)$ e a ocorrência de aborto em outras gestações (RP: 1,72; IC 95\% 1,08-2,74; $p=0,022$ ). Conclusão: Observou-se uma elevada prevalência de SD, comparando a estudos regionais, nas gestantes investigadas. Identificou-se como fatores associados ao desfecho, o estado civil e histórico de aborto, podendo trazer problemas na gestação e no pós-parto. Assim, percebe-se a necessidade de instrumentos e estratégias para identificar a presença de SD na fase inicial da gestação, para que sejam diagnosticados e tratados.

Palavras-chave: depressão; epidemiologia; fatores de risco; gravidez; atenção primária à saúde.

\begin{abstract}
Introduction: The onset of emotional disorders early in pregnancy is frequent, including depressive symptoms (DS) such as guilt, lack of appetite and energy. Objective: To measure the prevalence of depressive symptoms and associated factors in pregnant women attending primary care. Methods: Cross-sectional observational epidemiological study of pregnant women, who attend primary care in Caxias do Sul, Rio Grande do Sul, Brazil. Depressive symptoms were assessed using Patient Health Questionnaire (PHQ9). Gross analysis was performed, in which prevalence ratios (PR) and respective confidence intervals ( $\mathrm{Cl}$ 95\%) were calculated. The comparison of categorical variables occurred through the Chi-square test. The adjusted analysis was performed by Poisson regression, using the backwards technique, where the final model was constructed from the variables with $p \leq 0.20$ in the gross analysis. Results: The sample consisted of 76 pregnant women, of these $46.1 \%$ had depressive symptoms. The average age was 26.6 years $( \pm 5.95)$ and $72.4 \%$ were married or in a stable union. There was a significant association between depressive symptoms and marital status (PR: 1.54; 95\% Cl 1.00-2.37; $\mathrm{p}=0.045)$ and the occurrence of abortion in other pregnancies (PR: 1.72; 95\% Cl 1, 08-2.74; $p=0.022$ ). Conclusion: There was a high prevalence of depressive symptoms compared to regional studies in the pregnant women investigated. Factors associated with the outcome were marital status and history of abortion, which may cause problems during pregnancy and postpartum. Thus, there is a need for tools and strategies to identify the presence of depressive symptoms in early pregnancy, so that they can be diagnosed and treated.
\end{abstract}

Keywords: depression; epidemiology; risk factors; pregnancy; primary health care.

Recebido em: 10/01/2019

Revisado em: 13/05/2019

Aprovado em: 14/05/2019

Autor para correspondência: Rafaela Santi Dell'Osbel - Centro Universitário da Serra Gaúcha - Rua Os Dezoito do Forte, 2366 - CEP $95020-472$ - Caxias do Sul (RS), Brasil. E-mail: ra.fasanti@hotmail.com

Conflito de interesses: nada a declarar. 


\section{INTRODUÇÃO}

A gestação é uma fase fisiológica relevante na vida da mulher, ocorrendo diversas mudanças, entre elas, hormonais, psicológicas e sociais $^{1,2}$. Assim, necessitando de cuidados com a saúde, sendo fundamental a identificação e acompanhamento do estado psicológico e sintomas depressivos (SD) durante o período gestacional ${ }^{1,2}$.

O aparecimento de transtornos emocionais no início da gestação é frequente, junto a isso, são inúmeros os relatos de depressão ${ }^{3}$. Esta é conhecida por ser o transtorno mental mais comum, sendo a principal causa de deficiência no mundo e ocorrendo principalmente em mulheres ${ }^{4}$. De modo geral, os SD são semelhantes aos que ocorrem na depressão, como sentimento de culpa, falta de apetite e de energia $^{5,6,7,8}$. Na Atenção Básica, os SD devem ser rastreados ainda na primeira consulta de pré-natal ${ }^{9}$. Entretanto, sabe-se que na gestação a saúde mental não tem recebido a atenção necessária, provavelmente por ser associada à uma fase de bem-estar, também pelos SD apresentarem maior taxa de hospitalização no período de pós-parto ${ }^{10}$. Atualmente, destaca-se que a prevalência de SD em gestantes vem crescendo, variando entre $19,6 \%$ e 58,4\% $\%^{3,11,12,13}$. Assim, tornando-se necessários estudos e rastreio precoce desta população ${ }^{3}$.

Sabe-se que é comum a presença de SD no primeiro trimestre gestacional, logo, a presença deste distúrbio emocional pode trazer consequências para a gestante, como o aumento do risco para desenvolver depressão pós-parto ${ }^{10}$. Segundo estudo conduzido em gestantes no Canadá, grande parte das gestantes que apresentam depressão pós-parto já apresentavam depressão pré-natal ou SD durante a gestação, destas somente $6,6 \%$ foram identificadas como novos casos ${ }^{12}$. Percebe-se que os SD sofrem uma regressão ao longo dos trimestres gestacionais, tornando-os mais fortes e perceptíveis no início da gestação. Além disso, as consequências podem se estender ao recém-nascido, prejudicando o desenvolvimento do feto, acarretando redução no peso ao nascer, risco para parto prematuro e maiores chances de internações em unidades de terapia intensiva neonatal ${ }^{2,3}$.

Nesta perspectiva, existe risco de desenvolvimento de depressão durante o período gestacional e depressão pós-parto correspondente a presença dos SD, trazendo riscos tanto para a gestante quanto para o bebê․ Visto a magnitude do problema e possíveis consequências para gestante e recém-nascido e aos poucos estudos regionais encontrados, verifica-se a necessidade de novos estudos dirigidos a esta população, bem como a necessidade de verificar o rastreamento de SD e sua importância na Atenção Básica. Portanto, o presente estudo tem como objetivo medir a prevalência de sintomas depressivos e fatores associados em gestantes atendidas na Atenção Básica de Caxias do Sul/RS.

\section{MÉTODOS}

\section{Delineamento e população de estudo}

Realizou-se um estudo de delineamento epidemiológico observacional transversal, recorte do projeto de pesquisa
"Comportamentos de risco à saúde da gestante e o impacto na saúde materna e do recém-nascido na Atenção Básica de Saúde de Caxias do Sul-RS”.

\section{Característica amostral}

A cidade de Caxias do Sul dispõe de 46 Unidades Básicas de Saúde (UBS) na área urbana, sendo 24 UBS com Estratégia de Saúde da Família (ESF). Por critérios logísticos e limitações econômicas, optou-se por sortear apenas 16 UBS com ESF, número suficiente para garantir a representatividade de área total do município. Foram incluídas 76 gestantes que realizaram acompanhamento pré-natal em UBS na Atenção Básica de Caxias do Sul/RS, maiores de 18 anos, de primeiro trimestre gestacional (até 13 semanas de gestação) inscritas no SIS-Pré-Natal em cada uma das UBS sorteada. Gestantes analfabetas ou que não dominavam o português ou que apresentavam gestação de risco não foram incluídas do estudo.

\section{Coleta de dados}

Para a realização da coleta de dados, identificaram-se as gestantes de primeiro trimestre por meio de um contato prévio com as agentes comunitárias de saúde e enfermeiras responsáveis pelo rastreamento e acompanhamento das gestantes de cada UBS Posteriormente, entrou-se em contato com as gestantes de primeiro trimestre, usuárias das UBS sorteadas e inscritas no SIS-PréNatal, as quais foram convidadas para participar do estudo. As gestantes que aceitaram participar do estudo foram orientadas a comparecer na UBS para a aplicação do questionário. A aplicação do questionário e coleta de dados de prontuário foram realizadas por uma equipe de alunos dos cursos de graduação na área da saúde, previamente treinados, composta por 19 pessoas, no período de julho a outubro de 2017.

\section{Instrumento para coleta de dados}

Utilizou-se um questionário padronizado, pré-codificado, contendo instrumentos validados e questões elaboradas pelos pesquisadores. Foi realizado um estudo piloto com cinco gestantes de uma UBS sorteada, porém, estas não foram inclusas na amostra final. Destaca-se que uma parte do instrumento utilizado para a coleta de dados, constituída por questões comportamentais, foram obtidas por meio de um questionário autoaplicável.

O estudo incluiu variáveis socioeconômicas, demográficas, obstétricas, de estilo de vida, antropométricas e de comportamento alimentar. Referente aos aspectos socioeconômicos e demográficos (Tabela 1) foram investigadas as seguintes variáveis, idade (categorizada pelo valor da média em $\geq 27$; $\leq 26$ ), estado civil (casada/união estável; solteira/separada/divorciada), cor de pele autodeclarada (não branca; branca), número de pessoas que moram na casa (categorizada pelo valor da média em $\leq 3$ pessoas; $\geq 4$ pessoas), anos de estudo ( $\leq 10$ anos; $\geq 11$ anos), trabalho 
remunerado (sim; não) e nível socioeconômico (de A a C1; de C2 a E); A classificação do nível socioeconômico é feita por um sistema de pontuação, onde é avaliada a posse de itens, escolaridade da pessoa de referência e acesso a serviços públicos, a classe de pontos é dividida em: A (de 45 a 100), B1 (de 38 a 44), B2 (de 29 a 37), C1 (de 23 a 28), C2 (de 17 a 22), e D/E (de 0 a 16) ${ }^{14}$.

A variável obstétrica investigada foi o histórico de aborto (sim; não), coletada diretamente das informações do prontuário. Em relação às variáveis de estilo de vida, incluiu-se: tabagista (sim; não), tabagista anterior a gestação (sim; não), consumo de álcool (sim; não) e pratica atividade física semanalmente (sim; não). Considerou-se consumo de bebida alcoólica quando a gestante mantinha o habito de ingerir ao menos uma dose de bebida alcoólica por semana. Estabeleceu-se uma dose o equivalente a uma latinha de cerveja ou chopp $(330 \mathrm{ml})$ ou uma taça de vinho $(100 \mathrm{ml})$ ou uma dose de destilado $(30 \mathrm{ml})^{15}$. Referente a prática de atividade física, considerou-se aquelas que praticavam ao menos 150 minutos por semana ${ }^{16}$.

Quanto às medidas antropométricas, dados como o peso pré-gestacional e gestacional e a estatura foram coletados diretamente do prontuário, desta forma calculou-se as variáveis: Índice de Massa Corporal (IMC) pré-gestacional e atual. A classificação do IMC pré-gestacional é dividida em: baixo peso/desnutrido (menor que $18,5 \mathrm{~kg} / \mathrm{m}^{2}$ ), adequado/eutrófico (de 18,5 a $24,9 \mathrm{~kg} / \mathrm{m}^{2}$ ), sobrepeso (de 25,0 a $\left.29,9 \mathrm{~kg} / \mathrm{m}^{2}\right)$ e obesidade $\left(\geq 30,0 \mathrm{~kg} / \mathrm{m}^{2}\right.$ ) (eutróficas; não eutróficas). Já a variável IMC atual foi classificada de acordo com a semana gestacional, dividido em: baixo peso, adequado, sobrepeso e obesidade (adequado; não adequado $)^{17}$ (Tabela 2).
Referentes às variáveis de comportamento alimentar foram estudadas: insegurança alimentar (sim; não), imagem corporal (ausência de distorção; presença de distorção), compulsão alimentar e risco para transtornos alimentares (TA) (sem risco; com risco). Quanto à suplementação com ácido fólico, identificou-se a realização ou não da mesma (sim; não) (Tabela 2).

Como ferramenta para avaliação da insegurança alimentar foi utilizado a Escala Brasileira de Insegurança Alimentar (EBIA), tratando-se de uma escala com 14 perguntas de resposta "sim" ou "não"18. A imagem corporal foi avaliada segundo o Body Shape Questionnaire (BSQ), por meio de uma versão validada em português de Cordás e Castilho ${ }^{19}$, o qual permite verificar a insatisfação com a imagem corporal. Quanto a variável comportamento de risco TA, avaliou-se as seguintes práticas nos últimos três meses: compulsão alimentar (comer compulsivamente, com perda de controle); uso de laxantes; utilização de diuréticos; provocar vômitos; ficar sem comer ou comer pouco com o intuito de emagrecer. Considerando-se comportamento de risco para transtorno alimentar a gestante que apresentou qualquer um desses comportamentos por no mínimo uma vez por semana ${ }^{20}$ (Tabela 2).

\section{Variável desfecho - Sintomas depressivos}

Analisou-se através do Patient Health Questionnaire (PHQ9), tratando-se de um questionário padronizado e traduzido, composto por 9 perguntas referentes às últimas duas semanas ${ }^{21}$, são elas: 1) Quantos dias você teve pouco interesse ou pouco prazer em fazer as coisas?; 2) Quantos dias você se sentiu para baixo, deprimida ou sem perspectiva?; 3) Quantos dias você teve

Tabela 1: Variáveis socioeconômicas e demográficas em relação à prevalência de sintomas depressivos em gestantes atendidas na Atenção Básica de Caxias do Sul-RS. 2017 (n=76).

\begin{tabular}{|c|c|c|c|c|}
\hline Variáveis de exposição & $\mathbf{N}(\%)$ & Prevalência de SD* & RP $^{* \star}$ bruta (IC 95\%) & Valor de $p^{\star \star *}$ \\
\hline Idade & & & & 0,420 \\
\hline$\geq 27$ & $38(50,0)$ & 47,4 & 1 & \\
\hline$\leq 26$ & $38(50,0)$ & 54,1 & $1,20(0,76-1,88)$ & \\
\hline Estado Civil & & & & 0,063 \\
\hline Casada/União Estável & $55(72,4)$ & 44,4 & 1 & \\
\hline Solteira/Separada/ Divorciada & $21(27,6)$ & 66,7 & $1,50(0,97-2,30)$ & \\
\hline Cor da pele & & & & 0,420 \\
\hline Outras & $40(52,6)$ & 46,2 & 1 & \\
\hline Branca & $36(47,4)$ & 55,6 & $1,20(0,76-1,88)$ & \\
\hline № pessoas moram na casa & & & & 0,533 \\
\hline$\leq 3$ pessoas & $46(60,5)$ & 47,8 & 1 & \\
\hline$\geq 4$ pessoas & $30(39,5)$ & 55,2 & $1,15(0,73-1,80)$ & \\
\hline Anos de Estudo & & & & 0,917 \\
\hline$\geq 11$ anos & $34(44,7)$ & 50,0 & 1 & \\
\hline$\leq 10$ anos & $42(55,3)$ & 51,2 & $0,97(0,62-1,53)$ & \\
\hline Trabalho remunerado & & & & 0,706 \\
\hline Sim & $45(59,2)$ & 48,9 & 1 & \\
\hline Não & $31(40,8)$ & 53,3 & $1,09(0,69-1,71)$ & \\
\hline Nível socioeconômico & & & & 0,315 \\
\hline $\mathrm{De} A \mathrm{a} C 1$ & $24(31,6)$ & 54,9 & 1 & \\
\hline $\mathrm{De} C 2 \mathrm{a} \mathrm{E}$ & $52(68,4)$ & 41,7 & $0,75(0,44-1,30)$ & \\
\hline
\end{tabular}

*S: Sintomas Depressivos; **RP: Razão de prevalência; ***Valores em negrito apresentam significância estatística $(p \leq 0,05)$. 
dificuldade para pegar no sono ou permanecer dormindo ou dormiu mais do que de costume?; 4) Quantos dias a você se sentiu cansada ou com pouca energia?; 5) Quantos dias você teve falta de apetite ou comeu demais?; 6) Quantos dias você se sentiu mal consigo mesma ou achou que é um fracasso ou que decepcionou a sua família ou a você mesma?; 7) Quantos dias você teve dificuldade para se concentrar nas coisas (como ler o jornal ou ver televisão)?; 8) Quantos dias você teve lentidão para se movimentar ou falar (a ponto das outras pessoas perceberem), ou ao contrário, esteve tão agitada que você ficava andando de um lado para o outro mais do que de costume?; 9) Quantos dias você pensou em se ferir de alguma maneira ou que seria melhor estar morta?. Com as seguintes opções de resposta: nenhum dia, menos de uma semana, uma semana ou mais, e quase todos os dias. A classificação é gerada por meio do somatório dos pontos, podendo se encaixar nos seguintes critérios: ausência de SD $(\leq 4)$, SD leves (5$9)$, SD moderados (10-14), SD moderadamente severos (15-19) e SD severos $(\geq 20)^{22}$. Ainda assim, categorizou-se a variável em dicotômica pelo valor da média e mediana, presença de SD $(\geq 9)$ e ausência de $\mathrm{SD}(<9)^{23}$.

\section{Análises de dados}

A estruturação do banco de dados e análise estatística foi realizada por meio do programa SPSS Statistic Data 23, as análises bivariadas foram realizadas por meio do teste de qui-quadrado, para comparação de variáveis categóricas. A análise bruta foi realizada através do STATA 11 e calculou-se as razões de prevalência e respectivos intervalos de confiança em 95\% (IC95\%). Na análise ajustada realizou-se regressão de Poisson, utilizando o método de backwards, no qual as variáveis com significância de até $20 \%$

Tabela 2: Variáveis obstétricas, de estilo de vida, antropométricas e comportamento alimentar em relação à prevalência de sintomas depressivos em gestantes atendidas na Atenção Básica de Caxias do Sul, RS. 2017 ( $n=76)$.

\begin{tabular}{|c|c|c|c|c|}
\hline Variáveis de exposição & $\mathbf{N}(\%)$ & Prevalência de SD* & $\mathrm{RP}^{* \star}$ bruta (IC 95\%) & Valor de $p^{\star \star *}$ \\
\hline Tabagista & & & & 0,494 \\
\hline Não & $66(86,8)$ & 49,2 & 1 & \\
\hline Sim & $10(13,2)$ & 60,0 & $1,21(0,69-2,14)$ & \\
\hline Tabagista AG\# & & & & 0,268 \\
\hline Não & $47(61,8)$ & 45,7 & 1 & \\
\hline Sim & $29(38,2)$ & 58,6 & $1,28(0,82-1,99)$ & \\
\hline Consumo álcool & & & & $<0,001$ \\
\hline Não & $73(96,1)$ & 48,6 & 1 & \\
\hline Sim & $3(3,9)$ & 100,0 & $2,05(1,61-2,61)$ & \\
\hline IMC· pré-gestacional & & & & 0,571 \\
\hline Eutróficas & $36(47,4)$ & 53,8 & 1 & \\
\hline Não Eutróficas & $40(52,6)$ & 47,2 & $0,87(0,55-1,38)$ & \\
\hline IMC· atual & & & & 0,380 \\
\hline Adequado & $29(38,2)$ & 57,1 & 1 & \\
\hline Não adequado & $47(61,8)$ & 46,8 & $0,81(0,52-1,27)$ & \\
\hline Prática atividade física & & & & 0,746 \\
\hline Sim & $9(11,8)$ & 55,6 & 1 & \\
\hline Não & $67(88,2)$ & 50,0 & $0,90(0,47-1,70)$ & \\
\hline Histórico de aborto & & & & 0,035 \\
\hline Não & $59(77,6)$ & 44,8 & 1 & \\
\hline Sim & $17(22,4)$ & 70,6 & $1,57(1,03-2,40)$ & \\
\hline Ácido Fólico & & & & 0,732 \\
\hline Sim & $38(50,0)$ & 52,6 & 1 & \\
\hline Não & $38(50,0)$ & 48,6 & $0,92(0,58-1,45)$ & \\
\hline Insegurança alimentar & & & & 0,737 \\
\hline Não & $35(46,1)$ & 48,6 & 1 & \\
\hline Sim & $41(53,9)$ & 52,5 & $1,08(0,68-1,70)$ & \\
\hline Insatisfação corporal & & & & 0,575 \\
\hline Ausência de distorção & $61(80,3)$ & 49,2 & 1 & \\
\hline Presença de distorção & $14(18,4)$ & 57,1 & $1,16(0,68-1,96)$ & \\
\hline Compulsão alimentar & & & & 0,002 \\
\hline Sem risco & $43(57,3)$ & 34,9 & 1 & \\
\hline Com risco & $32(42,7)$ & 71,9 & $2,06(1,29-3,28)$ & \\
\hline Risco para $\mathrm{TA}^{\bullet}$ & & & & 0,003 \\
\hline Sem risco & $41(54,7)$ & 34,1 & 1 & \\
\hline Com risco & $34(45,3)$ & 97,1 & $2,06(1,27-3,34)$ & \\
\hline
\end{tabular}

*SD: Sintomas depressivos; **RP: Razão de prevalência; \#AG: Anterior a gestação; •IMC: Índice de massa corporal; •TA: Transtornos alimentares ***Valores em negrito apresentam significância estatística $(p \leq 0,05)$. 
na análise bruta $(\mathrm{p} \leq 0,20)$ ( 1 a 2$)$ foram incluídas no modelo final (Tabela 3). Considerou-se significância estatística $p \leq 0,05$, identificando associação entre SD e variantes de exposição.

\section{Aspectos éticos}

A pesquisa foi aprovada por um Comitê de Ética em Pesquisa, conforme a Resolução do Conselho Nacional de Saúde, n 466/12, sob n ${ }^{\circ}$ do parecer: 2.184.991. O Termo de Consentimento Livre e Esclarecido foi lido e assinado por todas as gestantes participantes do estudo, sob o qual ficou autorizada a participação, bem como, a divulgação dos resultados em anonimato.

\section{RESULTADOS}

A amostra foi constituída por 76 gestantes usuárias da Atenção Básica de Caxias do Sul/RS, destas 46,1\% apresentaram SD. Quanto à idade, a média encontrada foi de 26,6 anos ( $\pm 5,95 \mathrm{DP})$, variando de 18 a 41 anos. Em relação à cor da pele, um pouco mais da metade das gestantes se referiram como não brancas (52,6\%). A maioria das gestantes era casada ou em estavam em união estável $(72,4 \%)$, moravam com até três pessoas $(60,5 \%)$, tinham até 10 anos de estudo $(55,3 \%)$ e referiram ter um trabalho remunerado $(59,2 \%)$. No que diz respeito à renda, $68,4 \%$ pertenciam ao nível socioeconômico C2 a E (Tabela 1).

A tabela 2 apresenta as variáveis obstétricas, de estilo de vida, antropométricas e de comportamento alimentar, observou-se que a maioria $(86,8 \%)$ negou fumar na gestação, ao passo que, 38,2\% afirmaram ser fumantes antes do período gestacional. Entretanto, 96,1\% negaram o consumo de bebidas alcoólicas durante a gestação, $88,2 \%$ referiram não ter o hábito de realizar atividade física e $77,6 \%$ não apresentaram ocorrência de aborto em outras

Tabela 3: Análise da razão de prevalência ajustada em relação ao desfecho sintomas depressivos (SD) em gestantes atendidas na Atenção Básica de Caxias do Sul-RS. 2017 ( $n=76)$.

\begin{tabular}{|c|c|c|}
\hline Variáveis de exposição & $\begin{array}{l}\text { RP* ajustada }^{*} \text { (IC 95\%) }\end{array}$ & Valor de $\mathrm{p}^{\star *}$ \\
\hline Estado Civil & & 0,045 \\
\hline Casada/União Estável & 1 & \\
\hline Solteira/Separada/Divorciada & $1,54(1,00-2,37)$ & \\
\hline Consumo álcool & & 0,473 \\
\hline Não & 1 & \\
\hline Sim & $1,29(0,64-2,61)$ & \\
\hline Histórico de aborto & & 0,022 \\
\hline Não & 1 & \\
\hline Sim & $1,72(1,08-2,74)$ & \\
\hline Compulsão alimentar & & 0,482 \\
\hline Sem risco & 1 & \\
\hline Com risco & $1,70(0,38-7,60)$ & \\
\hline Risco para TA• & & 0,835 \\
\hline Sem risco & 1 & \\
\hline Com risco & $1,17(0,25-5,40)$ & \\
\hline
\end{tabular}

*RP: Razão de prevalência; •TA: Transtornos alimentares; **Valores em negrito apresentam significância estatística $(p \leq 0,05)$. Modelo final ajustado de acordo com a técnica de regressão de Poisson, variáveis $\operatorname{com} p \leq 0,20$ na análise bruta. gestações. Em relação às variáveis antropométricas, observou-se que 52,6\% das gestantes apresentavam IMC pré-gestacional classificando-as como não eutróficas. Em relação ao estado nutricional no momento da entrevista, $61,8 \%$ apresentaram-se classificadas como não adequado no IMC atual ${ }^{24}$. Ainda, $50 \%$ das entrevistadas realizavam a suplementação com ácido fólico e 53,9\% apresentaram insegurança alimentar. Em relação à imagem corporal e transtornos alimentares, observou-se que $18,4 \%$ apresentaram distorção da imagem corporal, $42,7 \%$ estavam com risco para transtorno de compulsão alimentar e 45,3\% foram identificados com comportamentos de risco para TA.

Em relação ao desfecho, as gestantes com 26 anos ou menos (RP: 1,20; IC 95\% 0,76-1,88; $p=0,420$ ), solteiras/separadas/divorciadas (RP: 1,50; IC 95\% 0,97-2,30; $p=0,063$ ), de cor de pele autodeclarada branca (RP: 1,20 ; IC 95\% 0,76-1,88; $p=0,420$ ) e com nível socioeconômico de A a C1 (RP: 0,75; IC 95\% 0,44-1,30; $p=0,315)$ apresentavam maior possibilidade de desenvolver SD (Tabela 1).

Mulheres tabagistas antes do período gestacional (21\%) (RP: 1,21; IC 95\% 0,69-2,14; $p=0,494)$, com hábito de consumir de bebida alcóolica (RP: 2,05; IC 95\% 1,61-2,61; $p=0,000$ ) e com histórico de aborto (RP: 1,57; IC 95\% 1,03-2,40; $p=0,035)$ apresentam mais probabilidade de desenvolver SD. Observa-se que o IMC pré-gestacional (RP: 0,87; IC 95\% 0,55-1,38; $p=0,571$ ) e o IMC atual (RP: $0,81$; IC $95 \% 0,52-1,27 ; p=0,380)$ não foram significativamente associados ao desfecho. Houve associação significativa com risco para TA (RP: 2,06; IC 95\% 1,27-3,34; $p=0,003$ ) e compulsão alimentar (RP: 2,06; IC 95\% 1,29-3,28; $p=0,002$ ) onde as participantes com risco para TA e compulsão alimentar apresentaram 2 vezes mais possibilidade de desenvolver SD. Ainda, percebe-se que a insegurança alimentar (RP: 1,08; IC 95\% 0,68-1,70; $p=0,737$ ) e a presença de distorção da imagem corporal (RP: 1,16; IC 95\% 0,68-1,96; $p=0,575$ ) estão fracamente associadas ao desfecho (Tabela 2).

Após a análise ajustada (Tabela 3) o estudo identificou associações significativas entre presença de SD e estado civil e a ocorrência de aborto em gestações anteriores. As gestantes solteiras/ separadas/divorciadas apresentaram $54 \%$ mais probabilidade de desenvolver o desfecho (RP: 1,54; IC 95\% 1,00-2,37; $p=0,045$ ) quando comparadas as casadas ou em união estável. Em relação ao histórico de aborto, observou-se prevalência $72 \%$ maior do desfecho (RP: 1,72; IC 95\% 1,08-2,74; $p=0,022$ ). Entretanto, identificou-se elevada prevalência de compulsão alimentar (70\%) (RP: $1,70 ; 0,38-7,60 ; p=0,482)$ para o desfecho quando comparado as gestantes que não apresentam compulsão alimentar, embora não tenha apresentado significância estatística.

\section{DISCUSSÃO}

O presente estudo teve como objetivo medir a prevalência de $\mathrm{SD}$ e fatores associados em gestantes de Caxias do Sul/RS, no qual 
se encontrou uma prevalência de $46,1 \%$ para o desfecho. A prevalência encontrada no presente estudo pode ser interpretada como um alerta para a comunidade investigada.

Quando comparada as prevalências encontradas em âmbito internacional percebe-se a importância de atentar aos SD durante o período gestacional. Estudo identificou níveis mais altos de SD no Brasil comparado a países desenvolvidos ${ }^{25}$. Em investigação realizada na Jamaica com 3.517 gestantes, observa-se que a prevalência nesta população $(19,6 \%)$ é inferior a encontrada neste estudo ${ }^{11}$. Ainda, em um estudo realizado no Canadá com 364 gestantes, a prevalência de SD durante a gestação foi de $28,3 \%{ }^{12}$. Por outro lado, em um estudo realizado em Melbourne/Austrália com 1102 gestantes, percebe-se uma prevalência de $58,4 \%{ }^{13}$. Logo, a prevalência assemelha-se a um estudo realizado na Arábia Saudita, onde a prevalência foi de $57,5 \%{ }^{26}$. Estes achados aproximam-se da prevalência aqui encontrada.

Em comparação a estudos nacionais e regionais, uma pesquisa realizada no Rio de Janeiro com 172 gestantes, houve prevalência de $33,7 \%$ de $\mathrm{SD}^{27}$. Ainda, em relação ao sul do país, em um estudo realizado em Pelotas com 4.130 gestantes, observa-se uma prevalência de SD de $16 \%{ }^{25}$. A grande maioria dos estudos apresentou prevalências menores comparadas ao presente estudo, acredita-se que devido as diferenças entre as populações e seus hábitos de vida.

Quando comparado as possíveis associações entre SD e as variáveis de exposição, o presente estudo apontou associações significativas a estado civil e histórico de aborto. Dessa forma, percebe-se que as gestantes solteiras/separadas/divorciadas apresentam maior probabilidade de desenvolver SD. Entretanto, sabe-se que a falta de intimidade nos relacionamentos está associada aos SD, assim, o ocorrido pode ser explicado pela falta de intimidade com um parceiro $^{28}$. Ainda, estudo identificou que gestantes que não coabitavam com seus parceiros apresentaram prevalência de $36 \%$ de $\mathrm{SD}^{25}$, onde consta que o aumento das preocupações durante o período gestacional está associado a $\mathrm{SD}^{28}$. Sugere-se que estas preocupações estejam possivelmente prejudicando e aumentado os SD nas gestantes solteiras/separadas/divorciadas, por sentirem-se sozinhas ou por falta de apoio.

Estudos apontam que mulheres com histórico de aborto apresentaram associação com os $\mathrm{SD}^{28,29}$. Ainda, segundo Costa et al. ${ }^{28}$, observa-se que preocupações com a gravidez também foram associadas ao desfecho. Entretanto, no presente estudo, percebe-se que as gestantes com histórico de aborto apresentaram $72 \%$ mais possibilidades de desenvolver SD. Acredita-se que essas probabilidades ocorrem devido ao aumento da preocupação com o período gestacional, baseado na possibilidade de ocorrer um novo aborto, interrompendo a gestação.

Observa-se elevada possibilidade para desenvolver SD (70\%) em gestantes com compulsão alimentar, apesar de não apresentar associação significativa. Entretanto, segundo Easter et al. ${ }^{30}$, que realizou um estudo com 137 mulheres, a compulsão alimentar apresenta associação com SD. Sugere-se que as gestantes com compulsão alimentar apresentem maiores preocupações com relação a gestação, seu peso e imagem corporal, tornando-as mais vulneráveis para transtornos psiquiátricos relacionados ao comportamento alimentar e SD.

Com relação à idade, o estudo identificou mulheres mais jovens $(\leq 26$ anos) com maior possibilidade (20\%) de desenvolver SD durante a gestação, indo na direção oposta a literatura observada ${ }^{2,25}$. No entanto, deve-se destacar que a associação não foi significativa e pode-se descartar potenciais variáveis de confusão, não mensuradas pelos pesquisadores.

No presente estudo observa-se que gestantes que não possuem trabalho remunerado possuem maior probabilidade de desenvolver o desfecho, apesar de não apresentar associação significativa. Resultados estes que assemelham-se aos encontrados em estudos ${ }^{12,29}$, onde as gestantes que não possuem trabalho remunerado apresentaram maiores possibilidades de desenvolver SD. Ainda, estudo associou a presença de SD à preocupações, estas podem estar associadas as preocupações financeiras ${ }^{13}$. Sugere-se que as gestantes que não possuem trabalho remunerado são expostas as preocupações e instabilidade financeiras, portanto tendem a desenvolver SD durante a gestação.

Identificou-se que o desfecho é predominante em gestantes com maior renda (25\%), embora não tenha apresentado associação significativa. De acordo com Bawahab et al. ${ }^{26}$, as preocupações financeiras estão associadas aos SD. Sugere-se que apesar da alta renda familiar, as preocupações financeiras estão presentes, devido ao número de moradores na residência e aos gastos gerados no período gestacional. Por esse motivo, acredita-se que a alta renda familiar associada às preocupações financeiras, sejam fatores de risco para o desenvolvimento de SD durante a gestação ${ }^{13}$.

Observa-se que as gestantes que possuíam o habito de fumar antes da gestação apresentaram maior prevalência em relação ao desfecho, apesar de não ser significativo. Estudos demonstraram que as gestantes que fumavam antes da gestação possuem menores chances de desenvolver depressão comparadas as que continuaram fumando no período gestacional ${ }^{31}$. Além disso, mulheres que param de fumar e desenvolvem SD tendem a ter recaídas ao tabagismo ${ }^{32}$. Sugere-se que os sintomas relacionados a uma possível abstinência ao tabaco estejam relacionados a maior presença de SD durante a gestação, assim, necessitando de mais atenção para a saúde psicológica da gestante durante este processo.

Com relação ao consumo de álcool, neste estudo observa-se que as gestantes que fazem uso de bebida alcóolica apresentam $29 \%$ mais possibilidades de desenvolver $\mathrm{SD}$, embora não apresente significância estatística na prevalência ajustada. De acordo com Leis et al. ${ }^{33}$, o uso de bebidas alcoólicas durante o período gestacional está associado aos SD, assemelhando-se aos resultados deste estudo. Segundo Davis et al..$^{34}$, mães com SD e depressão apresentam maior probabilidade de consumirem bebidas alcóolicas. Acredita-se que o uso de álcool agrave os SD, entretanto há possibilidade de as gestantes passarem a fazer uso do mesmo após o início dos SD. 
A insegurança alimentar é fortemente associada aos piores $\mathrm{SD}^{35}$. Neste estudo, a mesma aumenta em $8 \%$ a probabilidade de desenvolver o desfecho. Sugere-se que esteja associada a baixa renda, ao número de moradores da residência e ao aumento das preocupações com a alimentação durante o período gestacional ${ }^{2,28}$.

Em relação a distorção da imagem corporal, nota-se que a variável foi fracamente associada ao desfecho. Por outro lado, sabe-se que a distorção da imagem corporal é comumente associada à SD graves $^{36}$. Acredita-se que esse resultado tenha influência da idade gestacional, tratando-se do primeiro trimestre gestacional, das poucas mudanças com relação ao corpo e ao baixo ganho de peso ${ }^{37}$.

Como limitação deste estudo, destaca-se o viés de causalidade reversa, pois trata-se de um estudo transversal e não se considerou outros fatos que possam ter ocorrido influenciando os resultados. A homogeneidade da amostra, composta exclusivamente por gestantes do primeiro trimestre gestacional, o possível viés de memória, devido ao longo questionário que pode vir a confundir a gestante, além do reduzido número de entrevistadas. Ainda assim, são limitados os estudos nacionais que relacionem fatores de risco gestacional com SD.
Contudo, o presente estudo teve como potenciais a abrangência de UBS incluídas no estudo e os dados coletados, obtendo assim uma grande e diversificada quantidade de informações relevantes. Além disso, destaca-se o controle e cuidado para o tratamento dos dados, minimizando erros de digitação. Todos os participantes do estudo receberam treinamento e estavam capacitados para realizar a coleta, aumentando assim a veracidade dos dados coletados e dos resultados obtidos no presente estudo.

Conclui-se que os SD estão presentes em alguns casos na gestação, observando uma elevada prevalência, comparando a estudos regionais, nas gestantes atendidas pela Atenção Básica no munícipio. Ainda, identificou-se associação entre o desfecho com o estado civil e histórico de aborto, estes podem trazer problemas na gestação e no pós-parto. A alta prevalência encontrada neste estudo pode estar associada ao público entrevistado, a dependência do sistema único de saúde (SUS) para atendimentos e acompanhamento da gestação. Assim, percebe-se a necessidade de instrumentos e estratégias para identificar a presença de SD logo na fase inicial da gestação, para que os casos sejam diagnosticados e tratados.

\section{REFERÊNCIAS}

1. Aviram A, Hod M, Yogev Y. Maternal obesity: Implications for pregnancy outcome and long-term risks-a link to maternal nutrition. Int J Gynaecol Obstet. 2011;115:(Suppl 1):S6-10. http://doi.org/10.1016/S0020-7292(11)60004-0

2. Kingsbury AM, Plotnikova M, Najman JM. Commonly occurring adverse birth outcomes and maternal depression: a longitudinal study. Public Health. 2018;155:43-54 https://doi.org/10.1016/j.puhe.2017.11.001

3. González-Mesa ES, Arroyo-González ML, Ibrahim-Díez N, CazorlaGranados O. Mood state at the beginning of the pregnancy and its influence on obstetric and perinatal outcomes. J Psychosom Obstet Gynaecol. 2018:1-8.

https://doi.org/10.1080/0167482X.2018.1427726

4. Stewart BW, Wild CP. World cancer report 2014. Geneva: World Health Organization, 2014.

5. Jarde A, Morais M, Kingston D, Giallo R, MacQueen GM, Giglia L, et al. Neonatal outcomes in women with untreated antenatal depression compared with women without depression: a systematic review and meta-analysis. JAMA Psychiatry. 2016;73(8):826-37.

https://doi.org/10.1001/jamapsychiatry.2016.0934

6. McDonald S, Kehler H, Bayrampour H, Fraser-Lee N, Tough S. Risk and protective factors in early child development: Results from the All Our Babies (AOB) pregnancy cohort. Res Dev Disabil. 2016;58:20-30.

https://doi.org/10.1016/j.ridd.2016.08.010

7. Lewis A, Austin E, Galbally M. Prenatal maternal mental health and fetal growth restriction: a systematic review. J Dev Orig Health Dis. 2016;7(4):416-28.

https://doi.org/10.1017/S2040174416000076

8. Shakeel N, Eberhard-Gran M, Sletner L, Slinning K, Martinsen EW, Holme I, et al. A prospective cohort study of depression in pregnancy, prevalence and risk factors in a multi-ethnic population. BMC Pregnancy Childbirth. 2015;15:5

https://doi.org/10.1186/s12884-014-0420-0
9. Brasil. Instituto Sírio-Libanês de Ensino e Pesquisa. Protocolos da atenção básica: saúde das mulheres. Ministério da Saúde, 2016.

10. Almeida MS, Nunes MA, Camey S, Pinheiro AP, Schmidt MI. Mental disorders in a sample of pregnant women receiving primary health care in Southern Brazil. Cad Saude Pública. 2012;28(2):385-94. http://dx.doi.org/10.1590/S0102-311X2012000200017

11. Bernard O, Gibson RC, McCaw-Binns A, Reece J, CooreDesai C, Shakespeare-Pellington S, et al. Antenatal depressive symptoms in Jamaica associated with limited perceived partner and other social support: a cross-sectional study. PloS One. 2018;13(3):e0194338.

http://dx.doi.org/10.1371/journal.pone.0194338

12. Giallo R, Pilkington P, McDonald E, Gartland D, Woolhouse H, Brown S. Physical, sexual and social health factors associated with the trajectories of maternal depressive symptoms from pregnancy to 4 years postpartum. Soc Psychiatry Psychiatr Epidemiol. 2017;52(7):815-28.

http://dx.doi.org/10.1007/s00127-017-1387-8

13. Verreault N, Costa D, Marchand A, Ireland K, Dritsa M, Khalifé S. Rates and risk factors associated with depressive symptoms during pregnancy and with postpartum onset. J Psychosom Obstet Gynaecol. 2014;35(3):84-91

http://dx.doi.org/10.3109/0167482X.2014.947953

14. Associação Brasileira de Empresas de Pesquisa (ABEP). Critério de classificação econômica Brasil. CCEB 2008: Base LSE 2008. Disponivel em: http://www.abep.org/criterio-brasil. Acesso em: 10 ago 2016

15. World Health Organization (WHO). Self-help strategies for cutting down or stopping substance use: a guide. Geneva: WHO, 2010.

16. World Health Organization (WHO). Global recommendations on physical activity for health. Geneva: WHO, 2010.

17. Samur EA, Castillo C, Santoro RC, Aldea A. Propuesta de un nuevo estándar de evaluación nutricional en embarazadas. Rev Med Chile. 1997;125(12):1429-36. 
18. Corrêa AMS, Escamilla RP, Sampaio MFA, Marin-Leon L, Panigassi G, Maranha LK, et al. Relatório técnico: Acompanhamento e avaliação da segurança alimentar de famílias brasileiras: validação de metodologia e de instrumento de coleta de informação. Campinas: Unicamp, 2004.

19. Cordás TA, Castilho S. Imagem corporal nos transtornos alimentares: instrumento de avaliação: Body Shape Questionnaire. Psiquiatr Biol. 1994;2(1):17-21.

20. Ferreira JES, Veiga GV. Confiabilidade (teste-reteste) de um questionário simplificado para triagem de adolescentes com comportamentos de risco para transtornos alimentares em estudos epidemiológicos. Rev Bras Epidemiol. 2008;11(3):393-401. http://dx.doi.org/10.1590/S1415-790X2008000300006

21. Santos IS, Tavares BF, Munhoz TN, Almeida LSP, Silva NTB Tams BD, et al. Sensibilidade e especificidade do Patient Health Questionnaire-9 (PHQ-9) entre adultos da população geral. Cad Saúde Pública. 2013;29(8):1533-43. http://dx.doi.org/10.1590/0102-311X00144612

22. Kroenke K, Spitzer RL, Williams JB. The PHQ-9: validity of a brief depression severity measure. J Gen Intern Med. 2001;16(9):606-13. http://dx.doi.org/10.1046/j.1525-1497.2001.016009606.x

23. Manea L, Gilbody S, McMillan D. Optimal cut-off score for diagnosing depression with the Patient Health Questionnaire (PHQ-9): a meta-analysis. CMAJ. 2012;184(3):E191-6. http://dx.doi.org/10.1503/cmaj.110829

24. World Health Organization (WHO). Obesity: preventing and managing the global epidemic. Geneva: WHO, 2000.

25. Coll CVN, Silveira MF, Bassani DG, Netsi E, Wehrmeister FC, Barros FC, et al. Antenatal depressive symptoms among pregnant women: Evidence from a Southern Brazilian population-based cohort study. J Affect Disord. 2017;209:140-6. http://dx.doi.org/10.1016/j.jad.2016.11.031

26. Bawahab JA, Alahmadi JR, Ibrahim AM. Prevalence and determinants of antenatal depression among women attending primary health care centers in Western Saudi Arabia. Saudi Med J. 2017;38(12):1237-42

http://dx.doi.org/10.15537/smj.2017.12.21262

27. Pinto T, Vilela AA, Farias DR, Lepsch J, Cunha GM, Vaz JS, et al. Serum n-3 polyunsaturated fatty acids are inversely associated with longitudinal changes in depressive symptoms during pregnancy. Epidemiol Psychiatr Sci. 2017;26(2):157-68. http://dx.doi.org/10.1017/S204579601500116X

28. Costa ECV, Castanheira E, Moreira L, Correia P, Ribeiro D, Pereira MG. Predictors of emotional distress in pregnant women: the mediating role of relationship intimacy. J Ment Health. 2017:1-9. http://dx.doi.org/10.1080/09638237.2017.1417545

29. Alqahtani $\mathrm{AH}, \mathrm{Al}$ Khedair $\mathrm{K}$, Al-Jeheiman $\mathrm{R}$, Al-Turki HA, A Qahtani NH. Anxiety and depression during pregnancy in women attending clinics in a University Hospital in Eastern province of Saudi Arabia: prevalence and associated factors. Int J Womens Health. 2018:10:101-8.

http://dx.doi.org/10.2147//JWH.S153273

30. Easter A, Solmi F, Bye A, Taborelli E, Corfield F, Schmidt U, et al. Antenatal and postnatal psychopathology among women with current and past eating disorders: Iongitudinal patterns. Eur Eat Disord Rev. 2015;23(1):19-27. http://dx.doi.org/10.1002/erv.2328

31. Smedberg J, Lupattelli A, Mårdby AC, Øverland S, Nordeng $H$ The relationship between maternal depression and smoking cessation during pregnancy-a cross-sectional study of pregnant women from 15 European countries. Arch Womens Ment Health. 2015;18(1):73-84

http://dx.doi.org/10.1007/s00737-014-0470-3

32. Orton S, Coleman T, Coleman-Haynes T, Ussher M. Predictors of postpartum return to smoking: a systematic review. Nicotine Tob Res. 2018;20(6):665-73.

http://dx.doi.org/10.1093/ntr/ntx163

33. Leis JA, Heron J, Stuart EA, Mendelson T. Associations between depressive and anxious symptoms and prenatal alcohol use. Matern Child Health J. 2012;16(6):1304-11. http://dx.doi.org/10.1007/s10995-011-0892-8

34. Davis EC, Rotheram-Borus MJ, Weichle TW, Rezai R, Tomlinson M. Patterns of alcohol abuse, depression, and intimate partner violence among township mothers in South Africa over 5 years. AIDS Behav. 2017;21(Suppl 2):174-82. http://dx.doi.org/10.1007/s10461-017-1927-y

35. Maselko J, Bates L, Bhalotra S, Gallis JA, O'Donnell K, Sikander $S$, et al. Socioeconomic status indicators and common menta disorders: evidence from a study of prenatal depression in Pakistan. SSM Populat Health. 2018:4:1-9 https://doi.org/10.1016/j.ssmph.2017.10.004

36. Roomruangwong C, Kanchanatawan B, Sirivichayakul S, Maes M. High incidence of body image dissatisfaction in pregnancy and the postnatal period: Associations with depression, anxiety, body mass index and weight gain during pregnancy. Sex Reprod Healthc. 2017;13:103-9. https://doi.org/10.1016/j.srhc.2017.08.002

37. Vitolo MR. Nutrição: da gestação ao envelhecimento. 2ed. Rubio 2014. 